\title{
Impact of the Maternal and Child Health handbook in Angola for improving continuum of care and other maternal and child health indicators: study protocol for a cluster randomised controlled trial
}

Olukunmi Omobolanle Balogun ${ }^{1}$, Caroline Kaori Tomo ${ }^{1}$, Keiji Mochida ${ }^{2,3}$, Masashi Mikami', Henda da Rosa Vasconcelos ${ }^{4}$, Isilda Neves ${ }^{4}$, Hisakazu Hiraoka ${ }^{5}$, Hirotsugu Aiga ${ }^{5,6}$, Rintaro Mori ${ }^{7}$ and Kenji Takehara ${ }^{1 *}$ (D)

\begin{abstract}
Background: The Maternal and Child Health $(\mathrm{MCH})$ handbook is an integrated home-based record (HBR) designed to record in a single document all the information regarding health services provided to a pregnant woman and her child. The MCH handbook has the potential to support continuity of care which is key to strengthening maternal, newborn and child health. However, there is a lack of an integrated system to manage the health of pregnant women and young children on an ongoing basis in Angola. Thus, the Angolan Ministry of Health is partnering with the Japan International Cooperation Agency to build the capacity of healthcare providers through trainings and implementation of the $\mathrm{MCH}$ handbook to improve service utilisation. In this study, we will estimate the impact of an intervention package including distribution of $\mathrm{MCH}$ handbook and its supplementary interventions to women, on the utilisation of services provided at healthcare facilities from pregnancy through the postnatal and early childhood period.
\end{abstract}

Methods: This study is a cluster randomised controlled trial involving public healthcare facilities across all the municipalities located in Benguela Province, Angola. All women who go to participating healthcare facilities and with confirmed pregnancy around the beginning of the trial period will be included in the study. Women will be randomised according to the municipality where their primary maternity and/or childcare services are located. The intervention package will consist of MCH handbook distribution at all public healthcare facilities, MCH handbook utilisation training for healthcare providers and community mobilisation for women on the use of the MCH handbook. The intervention will be administered to all women in the intervention arm while those in the control arm will continue the traditional use of two stand-alone HBRs. The primary outcome measure for this study is to compare the proportion of women who achieve a complete continuum of care in both study arms.

(Continued on next page)

\footnotetext{
* Correspondence: takehara-k@ncchd.go.jp

${ }^{1}$ National Center for Child Health and Development, Tokyo, Japan

Full list of author information is available at the end of the article
}

C C The Author(s). 2020 Open Access This article is licensed under a Creative Commons Attribution 4.0 International License, which permits use, sharing, adaptation, distribution and reproduction in any medium or format, as long as you give appropriate credit to the original author(s) and the source, provide a link to the Creative Commons licence, and indicate if changes were made. The images or other third party material in this article are included in the article's Creative Commons licence, unless indicated otherwise in a credit line to the material. If material is not included in the article's Creative Commons licence and your intended use is not permitted by statutory regulation or exceeds the permitted use, you will need to obtain permission directly from the copyright holder. To view a copy of this licence, visit http://creativecommons.org/licenses/by/4.0/. The Creative Commons Public Domain Dedication waiver (http://creativecommons.org/publicdomain/zero/1.0/) applies to the data made available in this article, unless otherwise stated in a credit line to the data. 
(Continued from previous page)

Discussion: The findings from the study are expected to form a basis for revising the current trial version of the Angola $\mathrm{MCH}$ handbook and provide a framework for policy guiding nationwide scale-up and distribution of the MCH handbook.

Trial registration: ISRCTN Registry ISRCTN20510127. Registered on 4 June 2019

Keywords: Continuum of care, Service utilisation, MCH handbook, Maternal and child health outcomes, Angola

\section{Background}

The Republic of Angola, after almost 30 years of civil war, has made substantial economic and political progress in recent years [1], and such changes in the political, social and economic development of Angola directly affect population health. While the development of the health sector has not been universal within the country, the government and its partners are making continuous effort at improving access to health services [2]. The National Health System is today a landmark of a social policy geared towards human development [3], but there is still a long way to go. Health indicators are yet to reach adequate standards, and women and children's health continues to suffer. Maternal health is an important indicator in determining the status of health in a country and maternal mortality remains high in Angola. According to the World Health Organization (WHO) estimates, approximately 477 maternal death occurred per 100,000 live births in 2015 [4], primarily due to preventable causes. Also, Angola remains one of the African countries with the highest burden of under-5 (81 deaths per 1000 live births) and infant (54 deaths per 1000 live births) mortality rates, despite a consistent decreasing trend in recent years [5]. Some of the factors contributing to the poor maternal and child health indicators in Angola include weak health management systems [6] and the lack of an integrated system to manage the health of pregnant women and young children on an ongoing basis.

Towards rebuilding and strengthening the primary health care in Angola, its Ministry of Health $(\mathrm{MoH})$ with support from the World Bank formally launched the Municipal Health Service Strengthening Project and the campaign for the acceleration of maternal and infant mortality reduction in 2010 [7]. This involves providing universal access to a package of high-impact interventions, including antenatal care (ANC) and counselling for women, provision of insecticide-treated bed nets for pregnant women and children under five to prevent malaria, immunisations against vaccine-preventable childhood diseases, de-worming and delivery of essential micronutrients [1]. Care for women and children starting from pregnancy through childbirth into the babies' childhood has been promoted to improve maternal and child health through a continuum of care $(\mathrm{CoC})[8,9]$. Continuum of care involves the concept of an integrated system of care that guides and tracks mothers and babies over time, through a comprehensive array of health services involving all levels of intensity of care. Continuum of care for mothers, newborn and children forms the basis of health care in many high-income countries which also have the best maternal health indicators [8]. In contrast, health care is neither continuous nor integrated in many low-income countries, including Angola.

The Government of Angola recently developed the National Plan for Health Development 2012-2025 and prioritised pregnant women, infants and young children as the target population. With support from the United Nations (UN), Angola is also working to strengthen the health information system in the collection, processing and analysis of data [6]. A mechanism for attaining this goal involves an emphasis on strengthening institutional and management capacity, and capacity building for health workers through long- or short-term trainings. Given the political will, the Japan International Cooperation Agency (JICA), through its health sector cooperation, is providing support to the Government of Angola to enhance healthcare service delivery. Moreover, JICA is also supporting the $\mathrm{MoH}$ through its Technical Cooperation Project (TCP) "Project for Improving Maternal and Child Health Services through the implementation of the Maternal and Child Health (MCH) handbook".

The MCH handbook is an integrated home-based record (HBR) and is part of a scheme designed to record, in a single document, all the information and data regarding health services provided to and the health conditions of a mother and her child during the course of pregnancy, delivery and after birth, such as maternal care and the child's growth pattern and vaccination schedule $[10,11]$. In addition to other uses, the $\mathrm{MCH}$ handbook functions as a self-learning material, has the potential to reduce the need for multiple health records [12] and supports improvements in $\operatorname{CoC}[13,14]$. As a result, the $\mathrm{MCH}$ handbook has been attracting more attention from health ministries and professional organisations as an effective tool for promoting a life course approach to health care [11].

Despite the potentially important benefits of implementing the $\mathrm{MCH}$ handbook, there is a dearth of highquality studies to show its superiority to existing alternatives $[15,16]$. Therefore, this study will estimate the impact of an intervention package including distribution of 
$\mathrm{MCH}$ handbook and its supplementary interventions to women, compared with the traditional use of two standalone HBRs the on utilisation of services provided at healthcare facilities from pregnancy through the postnatal and early childhood period.

\section{Methods}

\section{Study objectives and design}

This study is a two-arm cluster randomised controlled trial (cRCT) to estimate the impact of a communitywide distribution of the $\mathrm{MCH}$ handbook and its supplementary interventions to women, compared with the traditional use of two stand-alone HBRs on $\mathrm{CoC}$ completion and various $\mathrm{MCH}$ outcomes. The unit of randomisation in this trial is the municipality (cluster). Specifically, this study will (1) evaluate the impact of $\mathrm{MCH}$ handbook on $\mathrm{CoC}$ completion, (2) assess uptake and utilisation of $\mathrm{MCH}$ handbook by both families and healthcare providers in Angola and (3) assess the impact of $\mathrm{MCH}$ handbook distribution and utilisation on maternal and child health.

\section{Study site}

The study will be carried out in Benguela Province, Angola. Benguela is situated in the west of the country. Benguela has 10 administrative divisions called municipalities and a population of approximately 2.6 million [17] - being the third most populous province in Angola. Its population is mostly ethnic Ovimbundu and the most widely spoken language besides Portuguese is Umbundo. The most populous municipalities in the province are Lobito, Benguela, Cubal and Ganda (in descending order) [17]. Agriculture, fisheries and livestock farming are the main economic activities of this population. Benguela was purposively selected for this study because of health indicators that are close to the national average, thus representing an ideal setting to show intervention impact on $\mathrm{MCH}$ outcomes and demonstrate feasibility for nationwide scaling up of $\mathrm{MCH}$ handbook in Angola.

\section{Cluster selection}

This impact evaluation study will be conducted in all 10 municipalities of Benguela Province. Each municipality comprises health communes that form the health administrative units for the province. The number and level of care provided by healthcare facilities vary between the municipalities. There are currently 209 public healthcare facilities [18] serving health needs and providing maternal newborn and child health $(\mathrm{MNCH})$ services across Benguela Province comprising 82 secondary- or tertiary-level hospitals and 127 primary healthcare units (Table 1). The primary healthcare level provides services including $\mathrm{MNCH}$ care and refers cases to secondary and tertiary healthcare level as needed.
Table 1 Number of healthcare facilities in Benguela Province

\begin{tabular}{|c|c|c|c|c|}
\hline \multirow[t]{2}{*}{ Arm } & \multirow[t]{2}{*}{ Municipality } & \multicolumn{2}{|c|}{ Level of care } & \multirow[t]{2}{*}{ Total } \\
\hline & & Primary & Secondary/tertiary & \\
\hline \multirow[t]{5}{*}{ Intervention } & Lobito & 24 & 3 & \multirow[t]{5}{*}{99} \\
\hline & Cubal & 20 & 2 & \\
\hline & Chongoroi & 16 & 1 & \\
\hline & Bocoio & 17 & 1 & \\
\hline & Balombo & 15 & 0 & \\
\hline \multirow[t]{5}{*}{ Control } & Benguela & 30 & 5 & \multirow[t]{5}{*}{106} \\
\hline & Catumbela & 16 & 1 & \\
\hline & Baia Farta & 15 & 0 & \\
\hline & Ganda & 24 & 3 & \\
\hline & Caibambo & 11 & 1 & \\
\hline Total & & 188 & 17 & 205 \\
\hline
\end{tabular}

There were 116,032 pregnant women in Benguela Province with 95,950 registered births in 2015. Of these, about $38 \%$ of women attended ANC services at a healthcare facility $\geq 4$ times [18]. Furthermore, ANC attendance varied by municipality whereby ANC service utilisation was highest in Benguela (60\%), Chongoroi $(48 \%)$ and Lobito (47\%) and lowest in Balombo (15\%) and Baia Farta (4\%) in 2015. In the same year, the rate of facility-based deliveries in Benguela Province was limited to $45 \%$, while $93 \%$ of children in the province were fully immunised [18]. Various factors, such as distance to the healthcare facility, affect women's utilisation of $\mathrm{MNCH}$ services across the province [19].

\section{Participant cohort}

The intervention will be administered to pregnant women through frontline providers of $\mathrm{MNCH}$ services in public healthcare facilities in Benguela. The impact of the intervention will be estimated by collecting data about maternal and newborn participants who seek care from participating facilities. All women pregnant at the beginning of the trial period and attending a public healthcare facility for $\mathrm{MNCH}$ services will be eligible for inclusion in the study. In order to ensure similar baseline characteristics among participant cohort across all municipalities, the study cohort will include all women confirmed to have become pregnant at the start of the trial implementation period. The participant cohort will be recruited at their first visit for any $\mathrm{MNCH}$ service consultation over the course of the study (Fig. 1). Based on the findings from a previous study [20], most pregnant women are expected to make their first visit to the healthcare facilities during the second trimester of pregnancy.

Prior to recruitment, study eligibility will be assessed and pregnant women who satisfy these criteria will be 


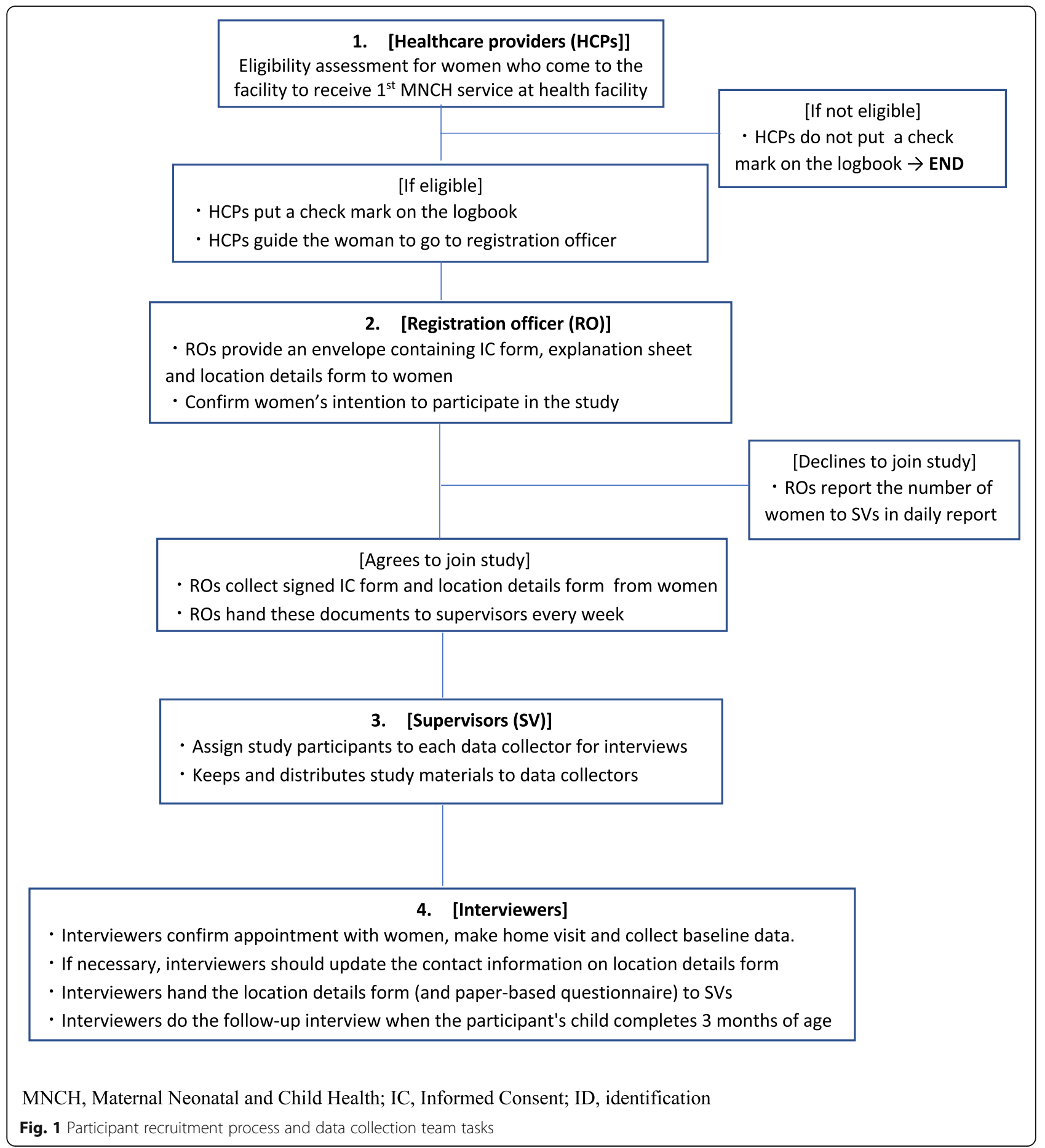

briefed about the trial and invited to participate. Consenting participants will then be registered and enrolled in the study. Figure 2 shows the study timeline according to the Standard Protocol Items: Recommendations for Interventional Trials (SPIRIT) diagram. For each registered participant, contact details will be collected in as many details as possible to enable follow-up home visits by the trial team. Written informed consent will be obtained from every participant joining the study prior to enrolment. Further, all participants will be assured of their rights to voluntary participation, privacy, confidentiality and free choice to withdraw from the study during the conduct of the trial in 


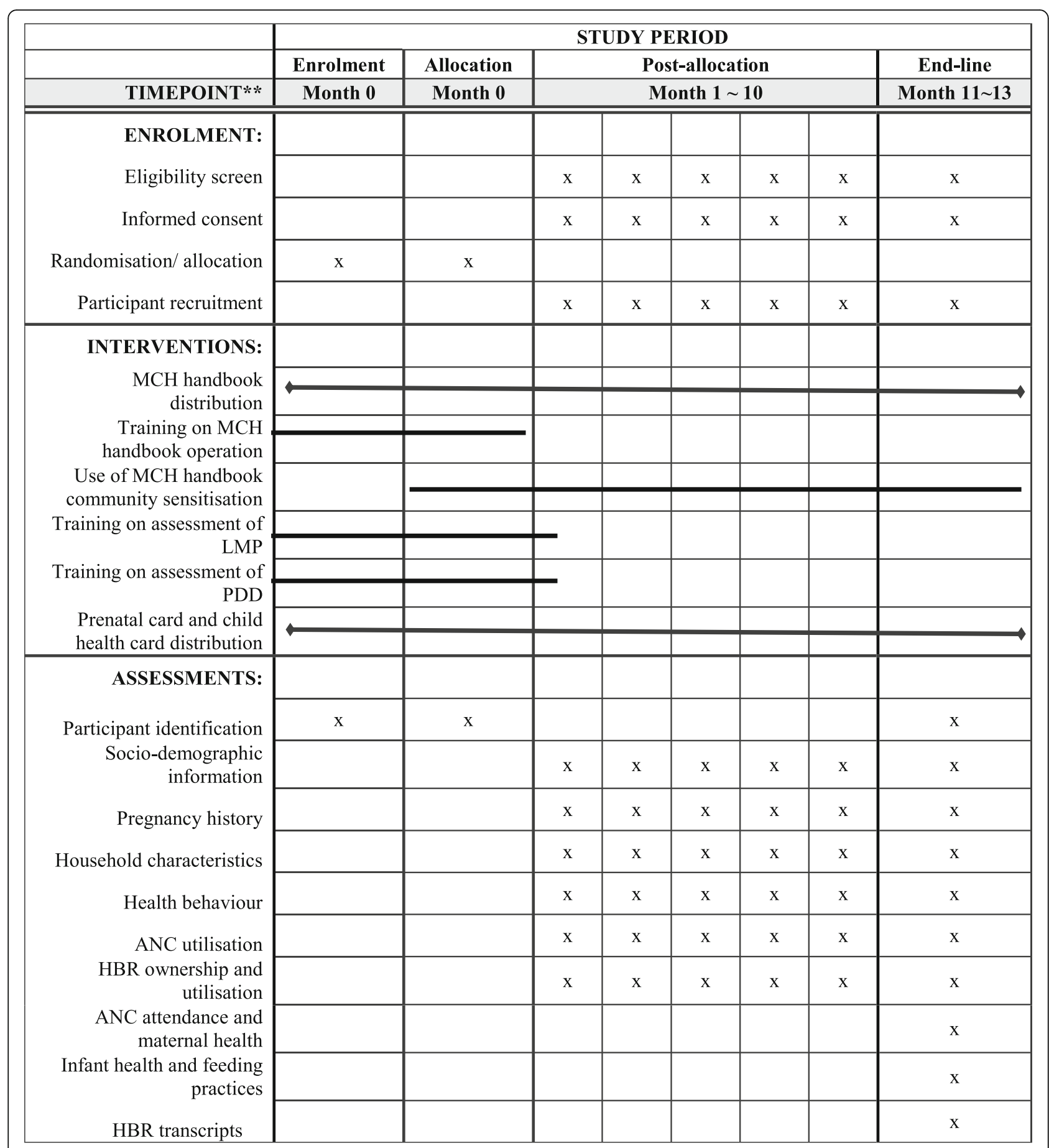

Fig. 2 schedule showing enrolment, allocation, intervention, baseline and end-line assessments. MCH, maternal and child health; LMP, last menstrual period; PDD, probable delivery date; ANC, antenatal care; HBR, home-based record

the process of obtaining informed consent (Fig. 3 in Appendix 1, Fig. 4 in Appendix 2).

\section{Eligibility criteria}

Participants will be eligible to join the study if they are confirmed by a healthcare provider to have become pregnant at the beginning of the trial period, had her last menstrual period (LMP) on any date from 1 March to 30 April 2019 or probable delivery date (PDD) on any date between 1 December 2019 and 31 January 2020. Participants will be selected among pregnant women and mothers within the defined cohort on their first visit 
to public healthcare facilities for $\mathrm{MNCH}$ services. Pregnant women will be excluded if they decline to participate in the study or are planning to move out of the study area while the impact evaluation study is ongoing.

\section{Power analysis}

This cRCT has three unit levels: the municipality, healthcare facility and the individual. A simulation-based power analysis was done in SAS software 9.4 (SAS Inc., Cary, NC, USA) using the generalised linear mixed model and taking these levels into consideration (Table 6 in Appendix 3). The power analysis was used to estimate the sample size needed to detect a $10 \%(25 \%$ in the intervention arm and $15 \%$ in the control arm) increase in the rate of $\mathrm{CoC}$ completion significant at $5 \%$ and assuming a $20 \%$ dropout rate. The intracluster correlation coefficients (ICC) used in this simulation-based power analysis were $0.010,0.015$ and 0.020 [21]; and assuming that the number of participants was 5000,7500 and 10 , 000 , the power ranged from 0.560 to 0.828 . We also confirmed in the simulation that type 1 error does not inflate. On the basis of these calculations, we aim to recruit at least 10,000 participants. The target sample size in each municipality will vary depending on the population size.

\section{Randomisation and allocation concealment}

This study is a two-arm study comprising an intervention arm and a control arm. The unit of randomisation in this trial is the municipality (cluster). Cluster randomisation was chosen due to the nature of the intervention and to minimise logistical and scientific difficulties associated with individual randomisation in large-scale longitudinal studies-such as contamination. Benguela Province consists of $10 \mathrm{mu}-$ nicipalities of varying sizes-Baia Farta, Balombo, Benguela, Bocoio, Caimbambo, Catumbela, Chongoroi, Cubal, Ganda and Lobito. All 10 municipalities will be included in the study. Population size and basic characteristics of each of the 10 municipalities were taken into consideration during the randomisation process to ensure a balance of socio-demographic characteristics between both arms.

Municipalities were allocated to either intervention or control arms using block randomisation $[22,23]$ by an experienced statistician in two steps. To ensure a balance between the two study arms, we ordered the municipalities by population size then applied block randomisation as described by Gattellari et al. [24]. First, municipalities were sorted by size based on the 2014 population data provided by the National Institute of Statistics [25]. Using the data, five blocks comprising of two municipalities each were created in the order of population size. Second, municipalities were randomly allocated either to the intervention or to the control arm within each block using computer-generated results. In this step, a pair of 4-digit code assigned to each block was fed into a SAS program that automatically returned a response, assigning one of the municipalities in the block to the intervention or control arm. The statistician was unable to predict assignment to either study arm. Municipalities allocated to the intervention or control arms were revealed simultaneously to the study team and municipal authorities at the kick-off meeting marking the start of the trial.

\section{Blinding (masking)}

Blinding will not be feasible due to the nature of the intervention; thus, neither participants nor trial personnel will be blinded in this study. However, the data analyst will be blinded to the allocation.

\section{Intervention}

The Angolan $\mathrm{MCH}$ handbook is a 36-page HBR designed to keep each pregnant woman's medical record from pregnancy through early childhood with educational information. It was developed based on the prenatal card and child health card currently being used in Angola, UNICEF educational materials, $\mathrm{MoH}$ documents and other materials [26]. The $\mathrm{MCH}$ handbook contains a log for making entries on maternal personal and health information during the course of pregnancy, delivery and postpartum; weight during and after pregnancy; infectious disease prevention and control; infant nutrition; child developmental milestones from the ages of 0-59 months; vaccination and illnesses records; and growth charts for children. The cultural appropriateness and acceptability of the handbook among pregnant women and healthcare providers have been previously assessed within a small cohort [27]. Even though the $\mathrm{MCH}$ handbook was generally well received among both mothers and healthcare providers, training for both groups would be required to derive the full benefits of the $\mathrm{MCH}$ handbook. Thus, the intervention consists of three basic components: (i) distribution of $\mathrm{MCH}$ handbook (the $\mathrm{MCH}$ handbook will be issued to pregnant women at each health facility at the HBR distribution points in the intervention arm), (ii) healthcare provider training on $\mathrm{MCH}$ handbook operation and (iii) community sensitisation and mobilisation among pregnant women on the use of the $\mathrm{MCH}$ handbook. Interventions will be delivered to pregnant women and healthcare providers in the intervention arm.

\section{Pregnant women/mothers}

The $\mathrm{MCH}$ handbook will be provided to the participant cohort during their first visit to receive $\mathrm{MNCH}$ services 
in each participating healthcare facility in the intervention arm. Explanation will be given on when and how to use the $\mathrm{MCH}$ handbook and on data recording. Participants will also receive health education based on the contents of the $\mathrm{MCH}$ handbook. Pregnant women and mothers in the study cohort attending healthcare facilities in control municipalities will continue to benefit from the traditional use of two stand-alone HBRs-the prenatal card and child health card. Pregnant women and mothers in both intervention and control municipalities will be asked to bring their $\mathrm{MCH}$ handbook or prenatal card and child health card to every occasion of $\mathrm{MNCH}$ service consultation. If a participant in either arm forgot to bring her handbook/card during a consultation, the attending healthcare provider will make a copy of the relevant recording page which will be used for keeping the record of the consultation and given to the participant.

\section{Healthcare providers}

Healthcare provider training on $\mathrm{MCH}$ handbook operation will be provided prior to distribution of the $\mathrm{MCH}$ handbook to pregnant women/mothers. They will be trained on skills needed for data recording into the $\mathrm{MCH}$ handbook, how to explain the use of the $\mathrm{MCH}$ handbook to pregnant women/mothers, make entries into the $\mathrm{MCH}$ handbook during regular consultations with pregnant women/mothers and on how to interpret data entries from the $\mathrm{MCH}$ handbook. Healthcare providers in the control arm will be trained on interviewing techniques for determining the date of LMP and on methods for calculating PDD only (Table 2). Healthcare providers in both intervention arms will be re-trained periodically over the intervention period.

\section{Data collection}

Study data collectors will collect data regarding $\mathrm{MNCH}$ services participants received. All data will be collected using a structured questionnaire by interviewer- facilitated tablet-based face-to-face interviews or abstracted from HBRs $(\mathrm{MCH}$ handbook in intervention arm or prenatal card and child health card in the control arm). However, where there is no record of $\mathrm{MNCH}$ services received in HBRs, mother's report of service utilisation will be used in place of the missing information as previously reported by Croft et al. [28]. Data collection will be conducted at participants' home or at a location of her choice at two time points-at baseline and at 3 months postpartum. Table 3 shows the data collection timeline for each outcome measure.

The baseline survey will comprise eight sections designed to elicit responses on socio-demographic factors, household characteristics, pregnancy history, maternal health behaviour and HBR ownership and utilisation. Data regarding ANC attendance, maternal health behaviour, pregnancy outcomes, infant feeding practices and infant and maternal health will also be collected from women retrospectively during the follow-up survey at 3 months postpartum. Verbal responses obtained from mothers will be double-checked against record entries made in the HBRs. In cases where discrepancies occur between the mother's report and HBR records, the latter will be prioritised. Additionally, we will verify the quality of HBR records by referring to the facility logbook data collected from a randomly selected sub-sample of women.

Interviews and data abstraction will be carried out by data collectors. Data collectors were selected from among persons with medical training or experience working with patients and were trained in tablet-based questionnaire administration and data collection processes. Training of the data collectors consisted of an overview of the study objectives, detailed description of baseline and follow-up questionnaire items and interviewing techniques, and field practice. The baseline and follow-up questionnaires were pre-tested before the start of the study. A summary of data collection timeline and questionnaire items is shown in Table 3. Interview responses and data abstracted by each data collector will

Table 2 Description of interventions

\begin{tabular}{lll}
\hline Description & Intervention arm \\
\hline Distribution of MCH handbook & $\times$ \\
ToT on MCH handbook operation & $x$ \\
OJT on MCH handbook operation & $x$ \\
Assessment of LMP & $x$ & $x$ \\
Determining PDD & $x$ & $\times$ \\
Community sensitisation and mobilisation on the use of the MCH handbook & $x$ \\
\hline
\end{tabular}

ToT training of trainers (trainers included representatives from each healthcare facility in the intervention arm), $O J T$ on-the-job training for other healthcare providers, $L M P$ last menstrual period, $P D D$ probable delivery date 
Table 3 Data collection timeline for each outcome measure

\begin{tabular}{|c|c|c|c|c|}
\hline \multirow[t]{2}{*}{ Time point } & \multirow[t]{2}{*}{ Item } & \multicolumn{2}{|l|}{ Data source } & \multirow[t]{2}{*}{ Location } \\
\hline & & Survey form & $\mathrm{HBR}$ & \\
\hline \multirow[t]{7}{*}{ Baseline } & Participant identification & $x$ & & Healthcare facility \\
\hline & Socio-demographic information & $x$ & & Participants home \\
\hline & Pregnancy history & $x$ & $x$ & \\
\hline & Household characteristics & $x$ & & \\
\hline & Health behaviour & $x$ & & \\
\hline & ANC utilisation & $x$ & $x$ & \\
\hline & HBR ownership and utilisation & $x$ & $x$ & \\
\hline \multirow[t]{6}{*}{ Follow-up (3 months postpartum) } & Participant identification & $x$ & & Participants home \\
\hline & HBR ownership and utilisation & $x$ & $x$ & \\
\hline & Health behaviour & $x$ & & \\
\hline & ANC attendance and maternal health & $x$ & $x$ & \\
\hline & Infant health and feeding practices & $x$ & & \\
\hline & HBR transcripts & & $x$ & \\
\hline
\end{tabular}

HBR home-based record, ANC antenatal care

be uploaded to a secure database and stored in dedicated computers on an on-going basis. Verification checks will be done on uploaded records, and attempts will be made to ensure the completeness and accuracy of collected data.

\section{Outcome measures}

The primary outcome for this study is a measure $\mathrm{MNCH}$ service utilisation assessed via the proportion of women who achieved time-dependent maternal behaviour-based $\mathrm{CoC}$ completion as at 3 months postpartum. $\mathrm{MNCH}$ service utilisation and $\mathrm{CoC}$ completion among women in low- and middle-income countries have been found to be associated with many factors such traditional and familial beliefs, distance to healthcare facility $[29,30]$, health care cost [2931], maternal education [30], perceived quality of care $[29,31]$ and service availability [32]. Therefore, factors influencing $\mathrm{CoC}$ completion may depend either on maternal behaviour and characteristics (demand-side factors) or service availability (supply-side factors). In this study, we hypothesise that distribution and utilisation of the $\mathrm{MCH}$ handbook will lead to maternal behaviour change leading to improved health-seeking behaviour and $\mathrm{MCH}$ outcomes. Consequently, the primary outcome is a time-dependent maternal behaviour-based measure of $\mathrm{CoC}$ completion. $\mathrm{CoC}$ completion will be assessed among all pregnant women who were recruited to the study during their ANC attendance. Under this measure, a timedependent service utilisation-based $\mathrm{CoC}$ completion will be achieved when pregnant women receive
$\mathrm{MNCH}$ services including a minimum number of ANC visits, facility-based delivery, postnatal care (PNC) for mother and newborn, and child vaccinations at two time points-at birth and at 2 3 months of infant age. $\mathrm{MNCH}$ service utilisation assessed via the proportion of women who achieve timedependent service-based $\mathrm{CoC}$ completion as at 3 months postpartum will be assessed as a secondary outcome. Coverage under this measure would depend on supply-side factors, such as service availability. Thus, $\mathrm{CoC}$ completion will be achieved only when pregnant women/mothers have received all the required $\mathrm{MNCH}$ services as shown in Table 4. Details of other secondary outcomes to be assessed are presented in Table 4.

\section{Other secondary outcomes}

1) Rate of $\mathrm{MNCH}$ service utilisation-number of actual $\mathrm{MNCH}$ service used

2) Neonatal mortality-number of infant deaths within the first 28 days of life

3) ANC service utilisation-frequency of ANC service use in each arm

4) Facility-based delivery-number of facility-based deliveries in each arm

5) Infant health check-up-proportion of children who received infant health checks

6) Maternal morbidity and pregnancy complication detection rate-number of cases of specified disease conditions and pregnancy complications diagnosed by a health care provider 
Table 4 List of outcomes and definition of outcome indicators

\begin{tabular}{|c|c|}
\hline Outcome & Definition \\
\hline \multicolumn{2}{|l|}{ Primary outcome } \\
\hline $\begin{array}{l}\text { Complete CoC } \\
\text { (maternal } \\
\text { behaviour-based) }\end{array}$ & $\begin{array}{l}\text { Time-dependent composite outcome. } \\
\text { Complete CoC is the minimum number of ANC } \\
\text { visits + facility-based delivery + PNC for mothe } \\
\text { and newborn + child vaccinations at two time } \\
\text { points-at birth and at } 2 \sim 3 \text { months. Includes } \\
\text { women who attended ANC and other MCNH } \\
\text { services only. } \\
\text { Complete CoC }=1 \text { when all indicators }=1\end{array}$ \\
\hline
\end{tabular}

\section{Secondary outcomes}

Rate of $\mathrm{MNCH}$ service utilisation

\begin{tabular}{|c|c|}
\hline & $\begin{array}{l}\text { minimum number of service utilisation. } \\
\text { Estimate means for each arm }\end{array}$ \\
\hline $\begin{array}{l}\text { Complete CoC } \\
\text { (service-based) }\end{array}$ & $\begin{array}{l}\text { Time-dependent composite outcome. } \\
\text { Composite outcome including } \geq 4 \text { ANC visits }+ \\
\text { facility-based delivery }+ \text { PNC of mother and } \\
\text { newborn }+ \text { complete child immunisation up } \\
\text { to } 2 \sim 3 \text { months. }\end{array}$ \\
\hline
\end{tabular}

Neonatal mortality No. of deaths within the first 28 days of life compared to the total number of deliveries in each study arm.

$\mathrm{NM}=1$ when all indicators $=1$

ANC service utilisation

No. of women who receive $\geq 4$ ANC visits in each arm regardless of the timing of first ANC. Adequate $\mathrm{ANC}=1$ if no. of $\mathrm{ANC}$ services $=1$

No. of women who receive $\geq 8$ ANC visits in each arm regardless of the timing of first ANC. Adequate $\mathrm{ANC}=1$ if no. of $\mathrm{ANC}$ services $=1$

Facility-based delivery

Infant health check-up

No. of facility-based deliveries in each arm.

Presence of at least one HBR entry from baby-well clinic attendance.

\section{Type}

Indicators

Details
Binary

First ANC timing
No. of ANC services
Facility delivery
PNC_mother
PNC_child
Vaccinations at
birth and at 2
months infant age
No. of ANC services
Facility delivery
PNC_mother
PNC_child

Vaccination

Binary

First ANC timing

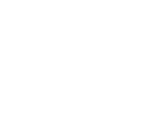

$\begin{array}{lll} & \text { Facility delivery } & \text { No }=0 ; \text { yes }=1 \\ & \text { PNC_mother } & \text { No HBR record }=0 ; \text { HBR record }=1 \\ & \text { PNC_child } & \text { No HBR record }=0 ; \text { HBR record }=1 \\ \text { Binary } & \text { Vaccination } & <7 \text { records }=0 ; 7 \text { records }=1 \\ & \text { Live birth } & \text { No }=0 ; \text { yes }=1 \\ & \text { Infant death } & \text { No }=0 ; \text { yes }=1\end{array}$

Timeline divided into $\leq 20,21 \sim 30$, $31 \sim 37$ and 38 weeks ga

$<4=0, \geq 4=1$ ( $\leq 20$ weeks); $<3=0$, $\geq 3=1$ ( $21-30$ weeks) $;<2=0, \geq 2=1$ (31 37 weeks); $<1=0, \geq 1=1$ (38 weeks)

$\mathrm{No}=0$; yes $=1$

No HBR record $=0$; HBR record $=1$ No HBR record $=0 ;$ HBR record $=1$ $<2$ vacc clinic visits $=0 ; 2$ vacc visits $=$ 1

No. of women with a minimum number of ANC in each arm

No of women with facility delivery in each arm

No. of women with mother PNC record in each arm

No. of women with child PNC record in each arm

No. of women with child vacc record in each arm

Timeline divided into $\leq 20,21 \sim 30$, $31 \sim 37$ and 38 weeks $\sim$ ga $<4=0, \geq 4=1$ ( $\leq 20$ weeks); $<3=0$, $\geq 3=1(21-30$ weeks); $<2=0, \geq 2=1$ (31 37 weeks); $<1=0, \geq 1=1$ (38 weeks)

No $=0$; yes $=1$

No HBR record $=0 ; H B R$ record $=1$

$<7$ records $=0 ; 7$ records $=1$
No. of ANC services 
Table 4 List of outcomes and definition of outcome indicators (Continued)

\begin{tabular}{|c|c|c|c|c|}
\hline Outcome & Definition & Type & Indicators & Details \\
\hline \multirow{10}{*}{$\begin{array}{l}\text { Maternal morbidity } \\
\text { and pregnancy } \\
\text { complication } \\
\text { detection rate }\end{array}$} & \multirow{10}{*}{$\begin{array}{l}\text { No. of cases of specified disease conditions } \\
\text { and pregnancy complications diagnosed by } \\
\text { an HCP in each arm. } \\
\text { Maternal morbidity }=1 \text { if any indicator }=1\end{array}$} & \multirow[t]{10}{*}{ Binary } & High blood pressure & No HBR record $=0 ;$ HBR record $=1$ \\
\hline & & & Preeclampsia & No HBR record $=0 ;$ HBR record $=1$ \\
\hline & & & Miscarriage & No HBR record $=0 ;$ HBR record $=1$ \\
\hline & & & Stillbirth & No HBR record $=0 ;$ HBR record $=1$ \\
\hline & & & Vaginal bleeding & No HBR record $=0$; HBR record $=1$ \\
\hline & & & Anaemia & No HBR record $=0 ;$ HBR record $=1$ \\
\hline & & & Malaria & No HBR record $=0 ;$ HBR record $=1$ \\
\hline & & & TB & No HBR record $=0 ;$ HBR record $=1$ \\
\hline & & & HIV & No HBR record $=0$; HBR record $=1$ \\
\hline & & & Diabetes & No HBR record $=0$; HBR record $=1$ \\
\hline \multirow{3}{*}{$\begin{array}{l}\text { Infant morbidity } \\
\text { rate }\end{array}$} & \multirow{3}{*}{$\begin{array}{l}\text { Total no. of disease cases attended by an HCP. } \\
\text { Infant morbidity }=1 \text { if all indicators }=1\end{array}$} & \multirow[t]{3}{*}{ Binary } & Past month sickness & No $=0 ;$ yes $=1$ \\
\hline & & & Sought treatment & $\mathrm{No}=0 ;$ yes $=1$ \\
\hline & & & Treatment from HP & No $=0 ;$ yes $=1$ \\
\hline \multirow[t]{2}{*}{ Infant mortality } & \multirow{2}{*}{$\begin{array}{l}\text { No of deaths of infants }<1 \text { year old compared } \\
\text { to the total number of deliveries in each study } \\
\text { arm. } \\
\mathrm{IM}=1 \text { when if both indicators = } 1\end{array}$} & \multirow[t]{2}{*}{ Binary } & Livebirth & No $=0 ;$ yes $=1$ \\
\hline & & & Infant death & $\mathrm{No}=0 ;$ yes $=1$ \\
\hline \multirow{6}{*}{$\begin{array}{l}\text { Maternal health } \\
\text { behaviour }\end{array}$} & \multirow{9}{*}{$\begin{array}{l}\text { Prevalence of maternal health behaviour } \\
\text { between arms with regard to tobacco use, } \\
\text { alcohol use, PMTCT, malaria prevention, use of } \\
\text { ITNs and family planning. Prevalence of each } \\
\text { indicator will be compared between the } \\
\text { intervention and control arms. }\end{array}$} & \multirow[t]{6}{*}{ Binary } & Current alcohol use & No $=0 ;$ yes $=1$ \\
\hline & & & Current tobacco use & $\mathrm{No}=0 ;$ yes $=1$ \\
\hline & & & РMTCT & $\mathrm{No}=0 ;$ yes $=1$ \\
\hline & & & Family planning use & $\mathrm{No}=0 ;$ yes $=1$ \\
\hline & & & $\begin{array}{l}\text { Knowledge change } \\
\text { tobacco use }\end{array}$ & $\mathrm{No}=0 ;$ yes $=1$ \\
\hline & & & $\begin{array}{l}\text { Knowledge change } \\
\text { alcohol use }\end{array}$ & No $=0 ;$ yes $=1$ \\
\hline \multirow[t]{3}{*}{ Malaria prevention } & & \multirow[t]{3}{*}{ Binary } & ITN possession & $\mathrm{No}=0 ;$ yes $=1$ \\
\hline & & & ITN use & No $=0 ;$ yes $=1$ \\
\hline & & & $\begin{array}{l}\text { Malaria prophylaxis } \\
\text { use }\end{array}$ & $\mathrm{No}=0 ;$ yes $=1$ \\
\hline $\begin{array}{l}\text { Maternal } \\
\text { depression }\end{array}$ & $\begin{array}{l}\text { No. of cases of maternal postnatal depressive } \\
\text { symptoms in each arm. }\end{array}$ & Binary & EPDS & $<10=0 ; \geq 10=1$ \\
\hline \multirow{3}{*}{$\begin{array}{l}\text { Infant feeding } \\
\text { practices }\end{array}$} & \multirow{3}{*}{$\begin{array}{l}\text { Appropriate infant feeding practices including } \\
\text { EIBF, EBF and absence of pre-lacteal feeding. } \\
\text { Adequate if all indicators }=1\end{array}$} & \multirow[t]{3}{*}{ Binary } & EIBF & After $1 \mathrm{~h}=0$; within $1 \mathrm{~h}=1$ \\
\hline & & & Pre-lacteal feeding & Yes $=0 ;$ no $=1$ \\
\hline & & & Current BF & $\mathrm{No}=0 ;$ yes $=1$ \\
\hline \multirow[t]{7}{*}{ Child vaccination } & \multirow{7}{*}{$\begin{array}{l}\text { Composite outcome for the no. of complete } \\
\text { fully vaccinated children at } 3 \text { months in each } \\
\text { study arm. } \\
\text { Vaccination = } 1 \text { if all indicators }=1\end{array}$} & \multirow[t]{7}{*}{ Binary } & Polio0 & No HBR record $=0 ;$ HBR record $=1$ \\
\hline & & & $\mathrm{BCG}$ & No HBR record $=0 ;$ HBR record $=1$ \\
\hline & & & Hepatitis B & N HBR record $=0 ;$ HBR record $=1$ \\
\hline & & & Polio1 & No HBR record $=0$; HBR record $=1$ \\
\hline & & & Pentavalent & No HBR record $=0 ;$ HBR record $=1$ \\
\hline & & & Pneumo1 & No HBR record $=0$; HBR record $=1$ \\
\hline & & & Rotavirus1 & No HBR record $=0$; HBR record $=1$ \\
\hline
\end{tabular}


Table 5 Minimum expected number of service utilisation to achieve complete CoC (primary outcome)

\begin{tabular}{llllllll}
\hline Time of first ANC (weeks) & ANC & Del & PNCm & PNCc & Vacc & Total & Complete CoC \\
\hline$\leq 20$ & 4 & 1 & 1 & 1 & 2 & 9 & 4 ANC, del., PNCm, PNCc, 2 vacc visits \\
$21 \sim 30$ & 3 & 1 & 1 & 1 & 2 & 8 & 3 ANC, del., PNCm, PNCc, 2 vacc visits \\
$31 \sim 37$ & 2 & 1 & 1 & 1 & 2 & 7 & 2 ANC, del., PNCm, PNCc, 2 vacc visits \\
$38 \sim$ & 1 & 1 & 1 & 1 & 2 & 6 & 1 ANC, del., PNCm, PNCc, 2 vacc visits \\
\hline
\end{tabular}

ANC antenatal care, del. facility delivery, $P N C m$ postnatal care for mother, PNCC postnatal care for child, vacc two vaccination clinic visits at birth and 2 months infant age

7) Infant morbidity rate-number of specific childhood disease cases attended by a health care provider

8) Infant mortality-number of infant deaths in each study arm

9) Maternal health behaviour-prevalence of maternal health behaviour between arms measured using at baseline and follow-up

10) Maternal depression-number of cases of maternal postnatal depressive symptoms

11) Infant feeding practices-prevalence of appropriate infant feeding practices in each study arm

12) Child vaccination - number of fully vaccinated children at the end of the study period

All outcomes will be assessed at follow-up except otherwise stated, and data will be collected through structured interviews and abstracted from HBRs.

The minimum number of $\mathrm{MNCH}$ services required for maternal behaviour-based $\mathrm{CoC}$ completion is shown in Table 5 and will depend on the timing of the first ANC visit and exposure to the intervention.

\section{Statistical analysis}

The intervention arm ( $\mathrm{MCH}$ handbook) will be compared to the control (traditional use of two standalone HBRs) in the main analysis. We will conduct descriptive analysis, and baseline data from the intervention and control arms will be analysed for comparability of baseline characteristics. Rates of missing data for each variable will be determined to assess the differences between the arms, and overall losses to follow-up will be determined. We will report reasons for losses within each arm and compare the reasons qualitatively.

We plan to measure intervention impact using two analysis sets: the intention-to-treat (ITT) set and the per-protocol analysis set (PPS). The ITT population will include all participants within the randomised municipalities analysed according to the municipality where they were first registered to receive $\mathrm{MNCH}$ services (i.e. discounting where they received second, third or fourth $\mathrm{MNCH}$ services) [33, 34]. The ITT population will be the primary analysis set, and all analyses will be conducted using this population unless otherwise stated. In the PPS, participant data will be analysed according to the treatment they actually received. Data from the participant with more than one MCNH service utilisation in the control arm will be included in the control arm regardless of the municipality of first registration for $\mathrm{MNCH}$ services in the PPS analysis.

The primary endpoint in this impact evaluation study is to compare the proportion of $\mathrm{CoC}$ completion achieved among mothers who received the $\mathrm{MCH}$ handbook in the intervention arm and those who received the traditional stand-alone HBRs in the control arm. We will evaluate the effect of the provision of $\mathrm{MCH}$ handbooks on $\mathrm{CoC}$ completion at the municipality level using a generalised linear mixed model (GLMM) assuming logit link and binomial distribution. All analyses will be based on complete case analysis. Regarding the number of healthcare service utilisations, the analysis will be conducted using survival analysis. We will also evaluate the intervention effects in a subgroup of participants based on socioeconomic status, urban/rural residence and other baseline characteristics while accounting for cluster effect.

All analyses will be done using STATA (version 13 or higher, Stata Press, College Station, TX).

\section{Losses to follow-up}

Participants who do not provide any baseline or follow-up data and those who withdraw their consent to participate in the study will be regarded as being lost to follow-up. To minimise such losses, a participant identification form will be used to collect each woman's contact information in as much detail as possible, including GPS location where baseline interview was conducted to facilitate repeat contact with mothers during the follow-up survey. The proportion of participants lost to follow-up will be compared between the two arms.

\section{Data management and storage}

All data collection processes will be standardised across the 10 municipalities. At each healthcare facility, 
pregnant women seeking $\mathrm{MNCH}$ services and eligible to join the study will be listed in the study register maintained by a data collector. Following enrolment, each woman will be given a unique identification number. A standardised tablet-based data collection form will be administered to each woman by trained study data collectors at baseline and follow-up. To ensure completeness of each record, virtually, all questions in the form are required, while at the same time ensuring participants right to voluntary participation. Completed forms will be uploaded to a secure server at the end of each day. The datasets will be password-protected and only accessible to the data manager.

We will not establish a third-party data monitoring committee due to the nature of the intervention and study design. However, periodic meetings involving all members of the study team will be held weekly to review the processes and progress related to the following: (1) participant recruitment and interviews; (2) completeness, accuracy and timeliness of uploading completed survey forms to secure server; and (3) any other logistical issues related to the study.

\section{Risk and benefit}

The intervention in this study is non-invasive and carries a less than minimal risk for adverse events in participating women. Consequently, potentially serious outcomes are not anticipated, and we do not envisage any reason, due to the intervention or study design to end the trial prematurely. Therefore, we will not conduct an interim analysis. Notwithstanding, disclosure of personal information and time taken for survey interviews may cause some discomfort to some participants. Data collectors will assure each participant of the anonymity and confidentiality of the data collected to make participants at ease. Adverse events that may arise will be assessed by the study team and reported to the responsible ethics committee. Reporting will include the type of event, circumstances, the extent of connection with study activities and the response provided. Potential benefits to participants in the study include improved $\mathrm{CoC}$ completion, knowledge of recommended health practices during pregnancy, recognition of danger signs and care of mother and newborn. Moreover, the intervention package will be delivered through the existing healthcare system for obtaining $\mathrm{MNCH}$ services.

\section{Discussion}

This is a study protocol for a cRCT aimed at estimating the impact of an intervention package-including the distribution of $\mathrm{MCH}$ handbook and its supplementary interventions to pregnant women, compared with the traditional use of two stand-alone HBRs on the utilisation of services provided at healthcare facilities from pregnancy through the postnatal and early childhood period. Few studies have shown that proper utilisation of HBRs could promote continuity of maternal and child health care $[14,16,35]$, and WHO recommends that each pregnant woman carries her own HBRs during pregnancy to improve continuity and quality of care [36]. Notwithstanding, the superiority of the integrated $\mathrm{MCH}$ handbook over other alternatives is an area of research in which there is a dearth of gold standard trials [15, 37]. We will attempt through this trial to provide higher quality evidence to demonstrate the benefits of implementing the $\mathrm{MCH}$ handbook over traditional stand-alone HBRs.

As a cluster-level intervention package, the trial employs a population-based approach to ensure uniform distribution of the $\mathrm{MCH}$ handbooks and its supplementary interventions. A cRCT is the preferred design in this trial to minimise logistical and scientific difficulties such as contamination between clusters and to optimise conditions to bring the intervention to scale within Angola in a real-life context. The trial's efficiencies lie in its design and data collection methods. The intervention package will be administered through the existing healthcare system for delivering $\mathrm{MNCH}$ services and with the support from the regional and local health divisions who routinely supervise the activities of frontline healthcare providers. Data will be collected through tablet-based interview methods. Mobile tablet devices have become an important tool for collecting primary data in clinical research, using a combination of functions including data entry, audio recorders, cameras, barcode readers and global positioning system receivers [38]. Using this method, the data management team in this trial will more easily collect and link data from the baseline and follow-up surveys. Mobile phones have also become an important tool for follow-up in public health research, through calls and text messaging [39, 40]. In order to reduce attrition rates, we will ask each participant to complete a participant identification form providing information to facilitate repeat contact $[39,41]$.

The findings from the impact evaluation study will be shared with the Angolan government and are expected to form a basis for revising the current trial version of the $\mathrm{MCH}$ handbook and for making an informed policy decision on nationwide scale-up and distribution of the $\mathrm{MCH}$ handbook. We plan to disseminate study findings through conference presentations and publication in international peer-reviewed journals.

This study protocol has been reported in accordance with the SPIRIT guidelines (Additional file 1) and including all items from the World Health Organization Trial Registration Data Set (Additional file 2).

\section{Trial status}

This article is the first protocol version. Participant recruitment started in June 2019 and is expected to be completed in June 2020. 


\section{Appendix 1}

IMPACT OF MATERNAL AND CHILD HEALTH HANDBOOK
EVALUATION

\section{EXPLANATION SHEET}

Hello. You are invited to participate in the impact evaluation study on home-based records (HBRs), which is being carried out in all municipalities in Benguela province.

Who is doing this study?

It is being caring out by TCHIKOS and Japanese International Cooperation Agency (JICA). The study is coordinated by the Nationa Directorate of Public Health (DNSP) and the Provincial Health Directorate in Benguela with partnership and funding from JICA.

If I agree to participate, what will happen?

Interviewers from TCHIKOS will visit you at home to ask you some questions about you and your baby's health and the living condition of your family. The interview will last approximately 30 minutes. This interview will be repeated soon after your baby is 2 or 3 months old.

Are they going to take my blood for analysis or do I have a risk in participating?

The interviewer will not take your blood or any other biological sample. They will only ask you questions and ask to see your HBR. Participating in this study is just meeting with the interviewer and honestly answering the questions that they ask you. This study does not involve some physical risk. The only discomfort is from answering some questions that you may consider personal.

What happens if I do not want to answer these personal questions?

Your participation is voluntary and the information you provide will be confidential. The interviewer will enter your responses in a device and send it directly to the TCHIKOS headquarters in Luanda, where there are information of other women like you. No one else will have access to this information. You will see that your name and other elements that personally identify you will not be sent. You are not required to answer questions that you do not want to answer, and you do not have to participate. But be aware that the study is extremely important for the Government to develop actions to support maternal and child health programs.

What is the purpose of this study?

The objective is to collect data that will inform the Government on how to work to improve maternal and child health services and status. The results of this study will be applied to all maternal and child health projects in the country. So, your participation is a privilege.

What are the benefits of participating in this study?

This study does not provide monetary nor assets benefits to the participants. In the long-term, your participation will benefit all women in Benguela and the country including your health and that of your children. The information you provide will tell the Government what to do to improve the health of women and children.

If after participation, I am interested to have more information?

You can call the numbers of our office that are at the end of this document for information. If you wish, you can also call Dr. Ernesto Ismael, 923511940 (Provincial Coordinator of this Study), Dr. Algimira Tomás, 923526553(Provincial Health Directorate), Dr. Maria Landinha, 923500529 (National Directorate of Public Health). You may also prefer to contact us physically at the address provided below.

When you receive this explanation sheet, you are confirming that you have received all the necessary information about the study provided by the registration officer in the hospital, you have agreed to participate by signing a form that has remained with him/her and provided all information about your residence to be located by the interviewer. If after a week you do not receive the interviewer's visit, please call or send a message to the numbers listed below.

Name of registration officer who assisted you: Date:

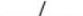

Fig. 3 Participant information sheet 


\section{Appendix 2}

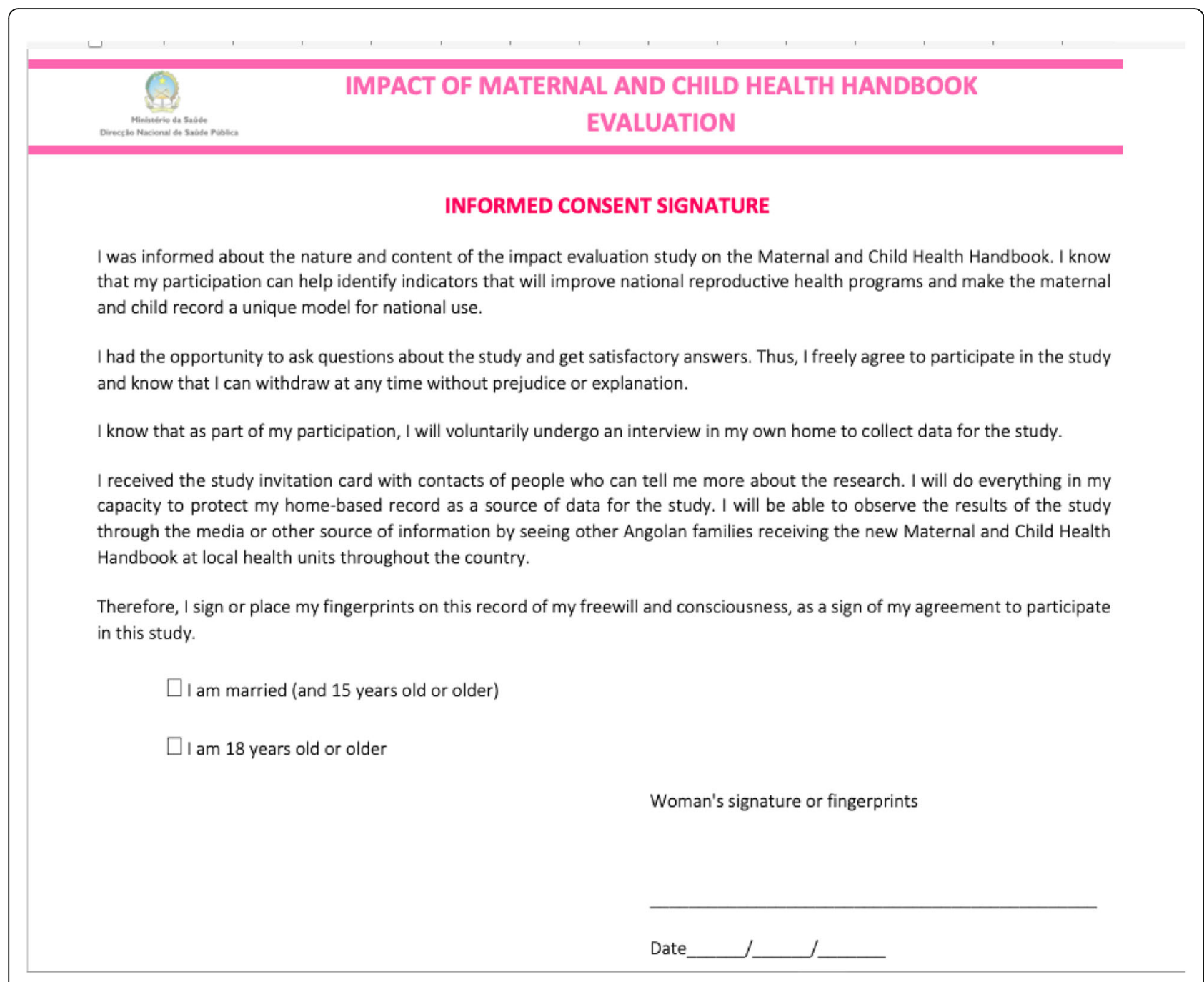

Fig. 4 Participant informed consent form

\section{Appendix 3}

Table 6 Sample size simulation

\begin{tabular}{|c|c|c|c|c|}
\hline \multicolumn{2}{|l|}{ Power } & \multicolumn{3}{|c|}{ Intervention, $25 \%$; control, $15 \%$} \\
\hline \multirow{2}{*}{\multicolumn{2}{|c|}{$\begin{array}{l}\text { Simulation: } 1000 \text { times } \\
a=0.05 \text {, two-sided }\end{array}$}} & \multicolumn{3}{|l|}{ Total $N$} \\
\hline & & 5000 & 7500 & 10,000 \\
\hline \multirow[t]{3}{*}{ ICC } & 0.010 & 0.776 & 0.808 & 0.828 \\
\hline & 0.015 & 0.639 & 0.658 & 0.674 \\
\hline & 0.020 & 0.560 & 0.570 & 0.605 \\
\hline
\end{tabular}




\section{Supplementary information}

Supplementary information accompanies this paper at https://doi.org/10. 1186/s13063-020-04664-w.

Additional file 1. SPIRIT Checklist: Recommended items to address in a clinical trial protocol and related documents.

Additional file 2. Items from the World Health Organization Trial Registration Data Set.

\section{Abbreviations}

ANC: Antenatal care; CoC: Continuum of care; CRCT: Cluster randomised controlled trial; DNSP: National Public Health Directorate; DSP: Directorate of Public Health; ICC: Intracluster correlation coefficient; ITT: Intention-to-treat: ISRCTN: International Standard Randomised Controlled Trials Number; JICA: Japan International Cooperation Agency; LMP: Last menstrual period; MCH: Maternal and Child Health; MoH: Ministry of Health; MNCH: Maternal newborn and child health; PDD: Probable delivery date; PNC: Postnatal care; PPS: Per-protocol analysis set; TCP: Technical Cooperation Project; UN: United Nations; UNICEF: United Nations Children's Fund; WHO: World Health Organization

\section{Acknowledgements}

The authors would like to recognise the contribution of various organisations and individuals to the trial. This study could only be possible through partnership and support provided by the National Public Health Directorate (DNSP) of the Ministry of Health in Angola in conceptualising the study and providing technical and logistical support. Preparations for the implementation of the study at the various study sites were facilitated by the Provincial Directorate of Public Health (DSP) in Benguela Province. The field surveys would not be possible without the support and commitment of the different team members from Domus Custodius holding TCHIKOS Consulting Agency and the local health authorities in Benguela Province. We would also like to highlight the valuable insights provided by Professor Stuart Gilmour into our study design and statistical analysis plan. We thank all the contributors to this study.

\section{Authors' contributions}

$K T, O O B, C K T, H A, H H, H R V, I N, R M, K M$ and MM all contributed to developing the concept and design of the study. KM, HA, HH, HRV and IN designed the intervention. $\mathrm{KT}, \mathrm{OOB}, \mathrm{CKT}, \mathrm{KM}, \mathrm{HA}, \mathrm{HH}, \mathrm{HRV}$ and IN provided general oversight during the design and implementation of the trial. KT, HA, $\mathrm{HH}$ and $\mathrm{RM}$ were responsible for securing funding for the study. $\mathrm{KT}$, OOB, $\mathrm{CKT}, \mathrm{KM}$ and $\mathrm{HRV}$ are responsible for the overall coordination of the trial conduct. $\mathrm{KT}, \mathrm{OOB}$ and $\mathrm{MM}$ contributed to the statistical analysis plan. OOB and CKT drafted the first manuscript. All authors read, approved, contributed to and provided critical review of the final manuscript.

\section{Funding}

The Angola Maternal and Child Health Handbook Implementation Research Project is supported by the Japan International Cooperation Agency (JICA). The funder had no role in the study design, data collection, decision to publish or preparation of this manuscript. The content herein is solely the responsibility of the authors and does not necessarily represent the official views of JICA.

\section{Availability of data and materials}

There are no data available in this article as no datasets were generated or analysed during the study protocol. The full datasets will be available (upon request) after they have been analysed and published.

\section{Ethics approval and consent to participate}

This trial protocol was approved by the Ethics Committee of the National Center for Child Health and Development (protocol identification No.1721) and the Ethics Committee of the Ministry of Health of the Republic of Angola (protocol identification No. 15/2018).
All eligible participants will be briefed on the study details prior to enrolment. Potential study participants will be asked to sign the informed consent form which will be returned to the interviewer. During the consent process, the objective, procedures, risks and benefits of the study will be explained to the participants and she will be encouraged to ask questions for clarifications. They will also be advised that their participation is voluntary and may choose not to participate in or withdraw her participation from the study at any time. Any participant who declines to join the study or who withdraws their participation in the study will be entitled to the same quality of care as study participants and treated without prejudice. Study participants will be given the assurance of anonymity and confidentiality of all collected information, before, during and after the trial. If an ancillary study is conducted, a signed informed consent will be obtained from every participant in the study if data collection/requests are not covered in the original informed consent process for the main Angola Maternal and Child Health Handbook Implementation Research Project.

Any protocol modifications will be communicated to the respective committees and the trial sponsors and registered on the ISRCTN.

\section{Consent for publication}

Not applicable

\section{Competing interests}

None declared. This document is not a formal publication of JICA, and the views expressed in this document by named authors are solely the responsibility of those authors.

\section{Author details}

${ }^{1}$ National Center for Child Health and Development, Tokyo, Japan. ${ }^{2}$ TA Networking Corp., Tokyo, Japan. ${ }^{3}$ Department of Global Health, Graduate School of Health Sciences, University of the Ryukyus, Nishihara, Japan. ${ }^{4}$ National Directorate of Public Health, Luanda, Angola. ${ }^{5}$ Japan International Cooperation Agency, Tokyo, Japan. ${ }^{6}$ School of Tropical Medicine and Global Health, Nagasaki University, Nagasaki, Japan. ${ }^{7}$ Graduate School of Medicine, Kyoto University, Kyoto, Japan.

Received: 3 May 2020 Accepted: 8 August 2020

Published online: 24 August 2020

\section{References}

1. UNICEF. Angola: background 2016. http://www.unicef.org/infobycountry/ angola_502.html. Accessed 7 June 2019.

2. Green A. Health in Angola in the wake of the presidential election. Lancet. 2017;390(10101):1477-8

3. Rodrigues SCC. Maternal mortality in Cabinda, Angola-description of the reproductive health care available; 2014

4. WHO, UNICEF, UNFPA, World Bank Group, the United Nations Population Division. Trends in maternal mortality: 1990 to 2015. Geneva: WHO; 2015.

5. UN-IGME. United Nations Inter-agency Group for Child Mortality Estimation (UN IGME) 2018. https://www.un.org/en/development/desa/population/ publications/mortality/child-mortality-report-2018.asp. Accessed 4 June 2019.

6. Unidas N. Quadro de Parceria entre o Governo de Angola e o Sistema das Nações Unidas (UNPAF) 2015-2019. Luanda, Angola; 2016.

7. The World Bank. Angola - municipal health service strengthening project 2010. http://www.worldbank.org/en/news/loans-credits/2010/06/08/angolamunicipal-health-service-strengthening-project. Accessed 24 May 2017.

8. Kerber KJ, de Graft-Johnson JE, Bhutta ZA, Okong P, Starrs A, Lawn JE. Continuum of care for maternal, newborn, and child health: from slogan to service delivery. Lancet. 2007;370(9595):1358-69.

9. de Graft-Johnson J, Kerber K, Tinker A, Otchere S, Narayanan I, Shoo R, et al. The maternal, newborn and child health continuum of care. Oppor Afr Newborns. 2006:23-36.

10. Osaki K, Aiga H. What is maternal and child health handbook? Tokyo: Japan International Cooperation Agency; 2016.

11. Osaki $\mathrm{K}$, Aiga $\mathrm{H}$. Adapting home-based records for maternal and child health to users' capacities. Bull World Health Organ. 2019;97(4):296.

12. Aiga H, Nguyen VD, Nguyen CD, Nguyen TT, Nguyen LTP. Fragmented implementation of maternal and child health home-based records in Vietnam: need for integration. Glob Health Action. 2016;9(1):29924. 
13. Bhuiyan SU, Nakamura Y. Continuity of maternal, neonatal and child health care through MCH handbook for ensuring the quality of life. In: $2008 \mathrm{MCH}$ handbook conference report Suita: Osaka University; 2009.

14. Osaki K, Hattori T, Kosen S. The role of home-based records in the establishment of a continuum of care for mothers, newborns, and children in Indonesia. Glob Health Action. 2013;6(1):20429.

15. Brown DW, Bosch-Capblanch $X$, Shimp L. Where do we go from here? Defining an agenda for home-based records research and action considering the 2018 WHO guidelines. Glob Health Sci Pract. 2019;7(1):6-11.

16. Magwood O, Kpadé V, Thavorn K, Oliver S, Mayhew AD, Pottie K. Effectiveness of home-based records on maternal, newborn and child health outcomes: a systematic review and meta-analysis. PLoS One. 2019; 14(1):e0209278.

17. Benguela Provincial Government. Perfil da Provincia 2015. http://www.benguela. gov.ao/InformacoesProvinciais.aspx?tipo=Perfil. Accessed 7 June 2019.

18. Direcção Provincial de Saúde do Governo de Benguela. Quadro das unidades sanitárias da província com os seguintes serviços referente ao ano de 2017. Benguela: Programa Provincial de Saúde Sexual e Reprodutiva; 2017.

19. Instituto Nacional de Estatística (INE), Ministério da Saúde (MINSA), Ministério da Planeamento e do Desenvolvimento Territorial (MPDT), and ICF. Key findings of the 2015-16 Angola IIMS. Luanda, Angola. Rockville, Maryland, USA: INE, MINSA, MPDT and ICF; 2017.

20. Nimi T, Fraga $S$, Costa D, Campos P, Barros H. Prenatal care and pregnancy outcomes: a cross-sectional study in Luanda, Angola. Int J Gynecol Obstet. 2016;135(Supplement 1):S72-S8.

21. Killip S, Mahfoud Z, Pearce K. What is an intracluster correlation coefficient? Crucial concepts for primary care researchers. Ann Fam Med. 2004;2(3):204-8.

22. Campbell MJ, Walters SJ. How to design, analyse and report cluster randomised trials in medicine and health related research. Hoboken: Wiley; 2014.

23. Ivers NM, Halperin IJ, Barnsley J, Grimshaw JM, Shah BR, Tu K, et al. Allocation techniques for balance at baseline in cluster randomized trials: a methodological review. Trials. 2012;13(1):120.

24. Gattellari M, Leung DY, Ukoumunne OC, Zwar N, Grimshaw J, Worthington JM. Study protocol: the DESPATCH study: delivering stroke prevention for patients with atrial fibrillation - a cluster randomised controlled trial in primary healthcare. Implement Sci. 2011;6(1):48.

25. (INE) INdE. Projecção da população província de Benguela. Luanda: INE; 2014

26. JICA. In: Agency JIC, editor. Japanese know-how supporting Angolan mothers and children. Tokyo: JICA; 2014.

27. JICA. Summary of ex-ante evaluation. Tokyo: Health Division 2 Human Development Department, JICA; 2016.

28. Croft TN, Marshall AM, Allen CK, Arnold F, Assaf S, Balian S. Guide to DHS statistics. Rockville: ICF; 2018.

29. Bohren MA, Hunter EC, Munthe-Kaas HM, Souza JP, Vogel JP, Gülmezoglu AM. Facilitators and barriers to facility-based delivery in low-and middleincome countries: a qualitative evidence synthesis. Reprod Health. 2014; 11(1):71.

30. Yeji F, Shibanuma A, Oduro A, Debpuur C, Kikuchi K, Owusu-Agei S, et al. Continuum of care in a maternal, newborn and child health program in Ghana: low completion rate and multiple obstacle factors. PLoS One. 2015; 10(12):e0142849.

31. Pell C, Meñaca A, Were F, Afrah NA, Chatio S, Manda-Taylor L, et al. Factors affecting antenatal care attendance: results from qualitative studies in Ghana, Kenya and Malawi. PLoS ONE. 2013;8(1):e53747.

32. Jani JV, De Schacht C, Jani IV, Bjune G. Risk factors for incomplete vaccination and missed opportunity for immunization in rural Mozambique. BMC Public Health. 2008;8(1):161.

33. Eldridge S, Kerry S. A practical guide to cluster randomised trials in health services research. Hoboken: Wiley; 2012.

34. Hollis $\mathrm{S}$, Campbell F. What is meant by intention to treat analysis? Survey of published randomised controlled trials. BMJ. 1999;319(7211):670-4.

35. Osaki K, Hattori T, Toda A, Mulati E, Hermawan L, Pritasari K, et al. Maternal and Child Health Handbook use for maternal and child care: a cluster randomized controlled study in rural Java, Indonesia. J Public Health. 2018; 41(1):170-82.

36. World Health Organization. WHO recommendations on antenatal care for a positive pregnancy experience. Geneva: World Health Organization; 2016.

37. World Health Organization. WHO recommendations on home-based records for maternal, newborn and child health. Geneva: World Health Organization; 2018.
38. Wilcox AB, Gallagher KD, Boden-Albala B, Bakken SR. Research data collection methods: from paper to tablet computers. Med Care. 2012:S68-73.

39. Booker CL, Harding S, Benzeval M. A systematic review of the effect of retention methods in population-based cohort studies. BMC Public Health. 2011;11(1):249.

40. Gaylor EE, Burnham MM, Goodlin-Jones BL, Anders TF. A longitudinal follow-up study of young children's sleep patterns using a developmental classification system. Behav Sleep Med. 2005;3(1):44-61.

41. Scott CK. A replicable model for achieving over $90 \%$ follow-up rates in longitudinal studies of substance abusers. Drug Alcohol Depend. 2004;74(1): 21-36.

\section{Publisher's Note}

Springer Nature remains neutral with regard to jurisdictional claims in published maps and institutional affiliations.
Ready to submit your research? Choose BMC and benefit from:

- fast, convenient online submission

- thorough peer review by experienced researchers in your field

- rapid publication on acceptance

- support for research data, including large and complex data types

- gold Open Access which fosters wider collaboration and increased citations

- maximum visibility for your research: over $100 \mathrm{M}$ website views per year

At $\mathrm{BMC}$, research is always in progress.

Learn more biomedcentral.com/submissions 